\title{
Measurement of Energy Expenditure in Elite Athletes Using MEMS-Based Triaxial Accelerometers
}

\author{
Andrew J. Wixted, Student Member, IEEE, David V. Thiel, Senior Member, IEEE, Allan G. Hahn, \\ Christopher J. Gore, David B. Pyne, and Daniel A. James, Senior Member, IEEE
}

\begin{abstract}
Fitness development and performance assessment of elite athletes requires an understanding of many physiological factors, many of these are direct and indirect measures of athlete energy expenditure. Many methods are physiological factor assessments and require the athlete to be constrained by laboratory equipment or periodic interruption of activity to take measurements such as blood samples are required to be taken. This paper presents a method that is entirely ambulatory and noninvasive, using microelectromechanical systems (MEMS) accelerometers. The commonly used output of commercial accelerometer-based devices (known as "counts") cannot discriminate activity intensity for the activities of interest. This, in conjunction with variability in output from different systems and lack of commonality across manufacturers, limits the usefulness of commercial devices. This paper identifies anthropometric and kinematic sources of inter-athlete variability in accelerometer output, leading to an alternate energy expenditure estimator based mainly on step frequency modified by anthropometric measures. This energy expenditure estimator is more robust and not influenced by many sources of variability that affect the currently used estimator. In this system, low-power signal processing was implemented to extract both the energy estimator and other information of physiological and statistical interest.
\end{abstract}

Index Terms-Accelerometer, biomechanics, energy expenditure, gait analysis, signal processing, sports engineering.

\section{INTRODUCTION}

$\mathbf{S}$ PORTS scientists monitoring the energy usage of elite athletes during training rely predominately on athlete self-monitoring augmented by monitored training sessions and traditional physiological measures such as heart rate and blood lactate concentration. Self-monitoring relies on the athlete recording their activities in a diary [1] and using a reference table to estimate energy expenditure [2]. Estimating athlete energy use during competition in field sports can be labor intensive and involves video monitoring and manual activity categorization. The availability of microelectronics devices and microelectromechanical systems (MEMS), particularly accelerometers provides an alternative, noninvasive method of automatic monitoring activity.

Manuscript received March 8, 2006, revised September 11, 2006, December 1,2006. This work was supported in part by the Cooperative Research Centre for Micro Technology and the Australian Institute of Sport. Expanded from a paper at the Sensors 2005 Conference. The associate editor coordinating the review of this manuscript and approving it for publication was Dr. Bahram Kermani.

A. J. Wixted, D. V. Thiel, and D. A. James are with the Centre for Wireless Monitoring and Applications, Griffith University, Nathan 4111, Brisbane, QLD Australia (e-mail: d.james@griffith.edu.au).

A. G. Hahn, C. J. Gore, and D. B. Pyne are with the Department of Physiology, Australian Institute of Sport, Bruce, ACT Australia.

Digital Object Identifier 10.1109/JSEN.2007.891947
The purpose of this study was to establish methods for the estimating of energy use of football (rugby) players in training and competition. MEMS accelerometers were chosen as the sensor platform because they are unobtrusive, have wide bandwidth, and can provide information pertaining to various activities of interest such as walking, running, athlete orientation, and impact contacts between athletes.

In most football codes, there is a high intensity of activity in the general proximity of the ball, although the dynamic and often chaotic nature of the play makes it difficult to easily characterize player movements. For the majority of the game time, most players are involved in relatively low intensity activity. This low intensity activity is interspersed with short bursts of high intensity activity followed by a recovery interval of variable duration. The predominant movement activities are: stationary, walking, jogging, running, sprinting, contacts (tackles, hit-ups, and other challenges). For physiological monitoring, both the duration at a particular intensity level and the temporal relationship between activities are important determinants of the overall physical load.

Due to the nature of competitive football, it is imperative that any monitoring device is as small and unobtrusive as possible. With the current state of microelectronics and MEMS development, the limiting factor on size and mass is the power source. By characterizing and interpreting the biomechanical activity as it relates to estimated energy expenditure, appropriate low-power signal processing algorithms have been developed. Simplified signal processing has reduced the processor load enabling a reduction in battery size.

\section{A. Accelerometers as Energy Estimators}

In 1960, Coates and Meade [3] correlated an individual's walking energy expenditure with the vertical distance of the trunk movement. There was a high correlation $(r=0.98)$ between volume of oxygen $\left(\mathrm{VO}_{2}\right)$ consumed and a factor consisting of the vertical displacement, the step frequency, and the subject's mass. In 1978, Reswick et al., [4] using a head mounted accelerometer, found that during walking, the integral of the vertical acceleration correlated with the lift per step and subsequently with $\mathrm{VO}_{2}$. Montoye et al. [5] (1983) developed a small portable uniaxial accelerometer and undertook a comparison between the accelerometer, $\mathrm{VO}_{2}$ and mercury switches. Subjects randomly worked through a series of physical activities chosen for their similarity to daily activities. The accelerometer output proved to be highly reproducible and, across the combined activities had moderate to high individual and group correlations with $\mathrm{VO}_{2}(n=21$, individual $r=0.63-0.89$, mean $r=0.79$, group $r=0.74$ ). 
Commercial devices became available in the 1990s and from this time onwards, the vertically mounted uniaxial accelerometer was widely used in studies of energy expenditure in free-living subjects. The more recent appearance of triaxial accelerometers has resulted in a range of studies comparing triand uniaxial accelerometers and various electronic pedometers, to evaluate their effectiveness as valid and reliable measures of energy expenditure [6]. The output of these commercial devices is a form of the integral of the vertical acceleration measured over a period of time and is commonly referred to as "counts." The counts output is not directly comparable across manufacturers [6], the counts output varies depending on the device location [7], and there can be considerable variation in counts from devices from the same manufacturer when tested on a testbed [8] or in the field [9]. Manufacturers may provide a conversion factor to convert from counts to calories or kilojoules.

For elite athletes and many recreational athletes, fitness training is graduated but traditional monitoring systems are often unable to discern the various levels of intensity outside of laboratory testing. Activities from a slow jog to sprinting may erroneously return the same accelerometry output leading to counts above a particular threshold simply being labeled "vigorous" (see [10]).

While various commercial uniaxial and triaxial accelerometry devices are available and group results may exhibit some correlation with energy expenditure, individual results can vary widely. For this research, it was originally hypothesized that the energy efficiency of a runner's technique may be discernable in the kinematic data from a triaxial accelerometer platform. Through experiments and analysis, we sought to establish whether variations in technique factor are responsible for the inter-subject variability shown in the acceleration integral.

\section{EXPERIMENTAL DESIGN}

Two separate treadmill studies were conducted in this project. First, ten male Australian football players walked and ran using their natural cadence at ten speeds on a motorized treadmill. These athletes were monitored by a triaxial accelerometer system fixed into a semi-elastic belt, fastened around the subject's waist. This placement ensured that the sensor was pressed into the L4-L5 medial lumbar vertebra region of the runner, a site approximating the subject's center of mass. The accelerometer axes were aligned with the vertical, mediolateral, and anterior-posterior axes of the subject. The second study involved ten mixed gender recreational athletes and was conducted in a similar manner to the first. In this study, the athletes were also monitored continuously during exercise for oxygen consumption $\left(\mathrm{VO}_{2}\right)$ using respiratory gas analysis from an open-circuit calometric system. Mass, height, and leg length of the athletes were recorded at the time of the tests.

\section{A. Hardware}

Two dual axis, $\pm 2 \mathrm{~g}$ MEMS analog devices accelerometers (ADXL202E [11]), tested as having an overload in excess of $\pm 7 \mathrm{~g}$, were mounted to the surface of a data acquisition module and aligned perpendicular to each other thereby creating a

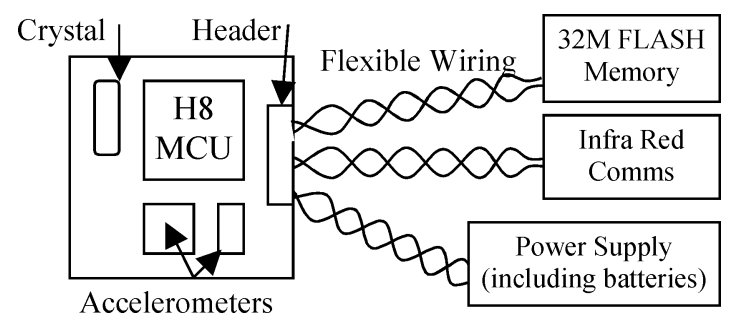

Fig. 1. Data acquisition system components.

triaxial accelerometer system. These devices were chosen for their availability, small dimensions $(5 \times 5 \times 2 \mathrm{~mm}$ die-size packaging) and long-term stability, requiring only a single calibration. Calibration of offset and sensitivity of the triaxial accelerometer system was performed using an inertial reference calibration technique proposed by Lai et al. [12]. The accelerometers formed part of a general-purpose sealed athlete monitoring system. This system comprised of a Hitachi H8 microcontroller based acquisition board running a low-power real-time operating system [13], Infrared (IR) communications circuitry and a power board with inductive charging circuits and batteries [14] (Fig. 1). The data acquisition board comprised of the minimal components necessary for the sampling system, so as to minimize the mass of this subsystem and the possible influence of this unit on the detected acceleration signal. Accelerometers were sampled at $150 \mathrm{~Hz}$ using the microcontroller's 10-bit analog-to-digital converters and data was logged to an internal 32-Mbyte flash memory card. At this sampling rate, the memory was sufficient for $16 \mathrm{~h}$ of raw data or approximately $48 \mathrm{~h}$ of compressed data. Up to ten separate training or competition sessions were recorded prior to downloading via the IR link. The motorized treadmill and the respiratory gas open-circuit calometric system were in-house custom built systems developed by the Physiology Department at the Australian Institute of Sport (AIS).

\section{ANALYSIS PHASES}

Analysis of the collected data occurred in several distinctive phases as the information was assimilated. Initial processing was performed to collect basic data and to confirm the operation of the system when compared to results from other systems in similar experiments. The second phase identified the ways in which individual results varied, using both the processed results and the raw signal data. The final analysis phase identified anthropometric or biomechanical correlates of the variation in accelerometry output. The understanding of the biomechanical contribution to the sensor data and its relationship to the physiological effects on the athlete allowed for the simplification of the signal processing necessary to extract the primary information of interest.

\section{A. Initial Processing}

Post-processing of the logged raw data was used to: confirm the functioning of the system, identify the factors that impacted the accelerometer signal, and to exploit the signal subsequently obtained. $\mathrm{VO}_{2}$ processing was performed as outlined in [15]. 


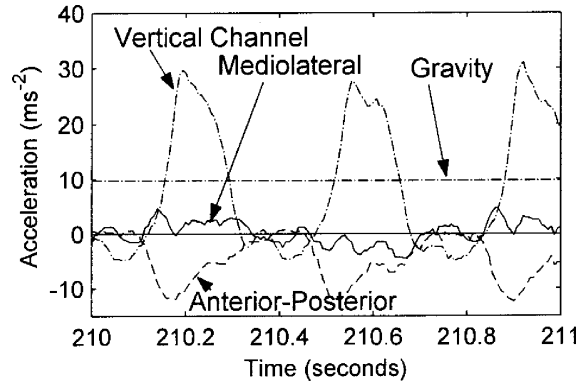

Fig. 2. Dynamic ambulatory signal for athlete running at $13 \mathrm{kmh}^{-1}$. The predominate signal is the vertical acceleration. Left-right asymmetry is apparent. Traces are individual to an athlete.

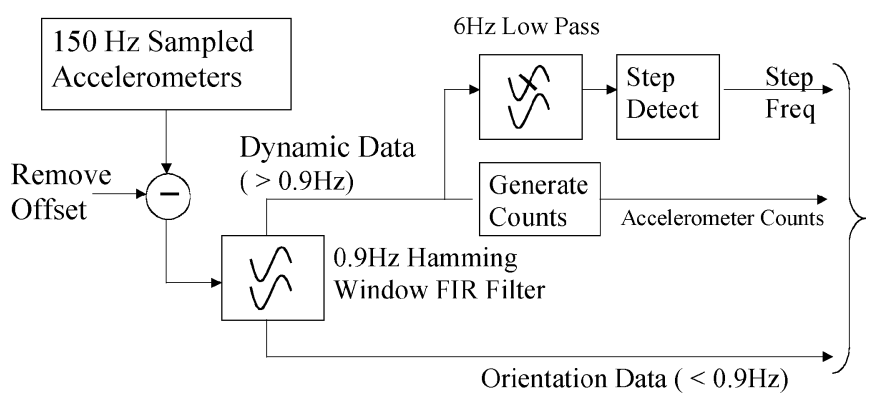

Fig. 3. Accelerometer processing flow diagram showing key outputs for further processing.

The raw triaxial acceleration signal was split into orientation and ambulatory signals. The orientation of the sensor was extracted using a low-pass $0.9-\mathrm{Hz}$ Hamming windowed finite-impulse response (FIR-H) filter. After subtracting the orientation signal, the remaining signal contained the dynamic ambulatory signal (Fig. 2). Using the orientation signal as input to a rotational tensor, the data was rotated to ensure the vertical axis had an average vertical orientation. Fig. 3 shows the process flow. Uniaxial energy estimators were generated for all three axes using the integral of the square of the acceleration signals (summing the square of the analog-to-digital converter output after removing the device offset and gravity) while the triaxial energy estimator used the integral of the sum of the squares of the signals from the three accelerometers. The estimate of energy expenditure was taken as a 10-s average from a period of steady-state walking or running at each speed. This energy estimator is referred to as "Accelerometer Counts." Step frequency was automatically estimated using the interval between successive positive zero crossings in the vertical acceleration. Step frequency was also manually calculated by counting the cycles in the vertical acceleration in the 10-s segment used for the above process. During walking at the lower speeds, for some athletes there was no clearly defined acceleration cycle. In these cases, steps were counted from a digital video recording of the test session.

1) System Confirmation Results: Results from this processing were similar to results from other studies such as [16], which combined high-speed treadmill running with uniaxial accelerometer and $\mathrm{VO}_{2}$ analysis. Some aspects of [6] were also comparable, this study compared a variety of accelerometer-based devices against $\mathrm{VO}_{2}$ but speeds only went to approximately $13 \mathrm{kmh}^{-1}$.

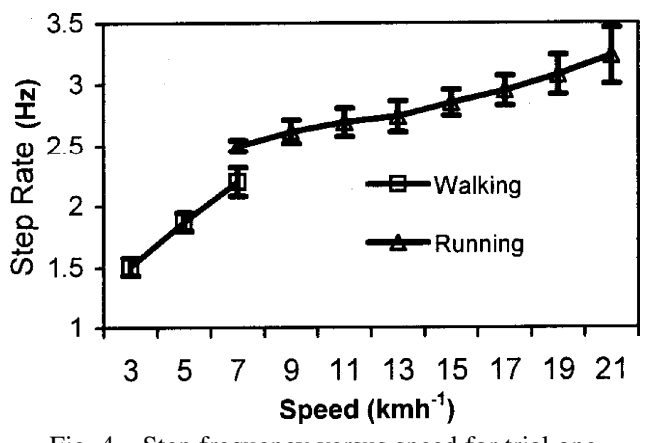

Fig. 4. Step frequency versus speed for trial one.

Regression analysis of speed against energy estimates from $\mathrm{VO}_{2}$ (corrected for the subject's mass), gave a correlation coefficient of $R^{2}=0.96$ ( $R^{2}=0.97$ in [16] and 0.98 in [6]). Triaxial and vertical-uniaxial accelerometer counts correlated with walking speed $\left(R^{2}=0.83, R^{2}=0.62\right)$. Uniaxial accelerometer counts at running speeds varied widely between individuals and gave no useful correlation with speed Fig. 5(a). During running, the standard deviations shown in Fig. 5(a) were approximately $22 \%$ of the mean for uniaxial counts and $15 \%$ of the mean for triaxial counts. The lowest individual uniaxial result over the running speeds $11-21 \mathrm{~km}^{-1}$ was $50 \%$ lower than the highest (approximately 2500 versus 5000 counts). For uniaxial counts—only one athlete recorded peak counts at $21 \mathrm{kmh}^{-1}$, most peaked at $15-17 \mathrm{kmh}^{-1}$. The uniaxial result of Fig. 5(a) is graphically similar to that of [16].

Triaxial accelerometer counts at running speeds gave a range of individual correlations with substantial inter-subject variation (Fig. 5(a) \& (b)). Triaxial counts were higher than the corresponding uniaxial counts and tended to give a more linear individual running response. The increased counts appeared to be due to capturing more of the athlete's activity, while the linear response appeared to be due to recapturing of vertical acceleration signal—otherwise lost as a result of biomechanical activity tilting the sensors and robbing signal from the vertical accelerometer.

Across both trials, step frequency had strong correlation with speed $\left(R^{2}=0.80, R^{2}=0.74\right.$ in [16]), with walking having a stronger step-frequency to speed correlation $\left(R^{2}=0.89\right)$ than running $\left(R^{2}=0.69\right)$. Individual results with running and walking treated separately were in the range $R^{2}=0.90-1.00$. Walking and running gave two distinctly different step-frequency to speed responses (Fig. 4).

For individuals, step-frequency correlated highly with massadjusted $\mathrm{VO}_{2}$ energy estimates $\left(R^{2}=0.80-0.99\right)$. In contrast, however, the group results were far weaker $\left(R^{2}=0.29\right)$.

2) Experimental Observations: During this process, these observations were noted.

1) Using an effectively unlimited sensor bandwidth, impulse signals exceeded the limits of this device $( \pm 7 \mathrm{~g})$. These spikes, together with an unknown sensor recovery time, make accelerometer counts unreliable. The high-amplitude signal was confirmed using higher rated sensors. It is not known how, or if, this impulse signal affected other accelerometer-based, monitoring devices although this question is raised in [16], commenting that the acceleration measured by force plates exceeded 

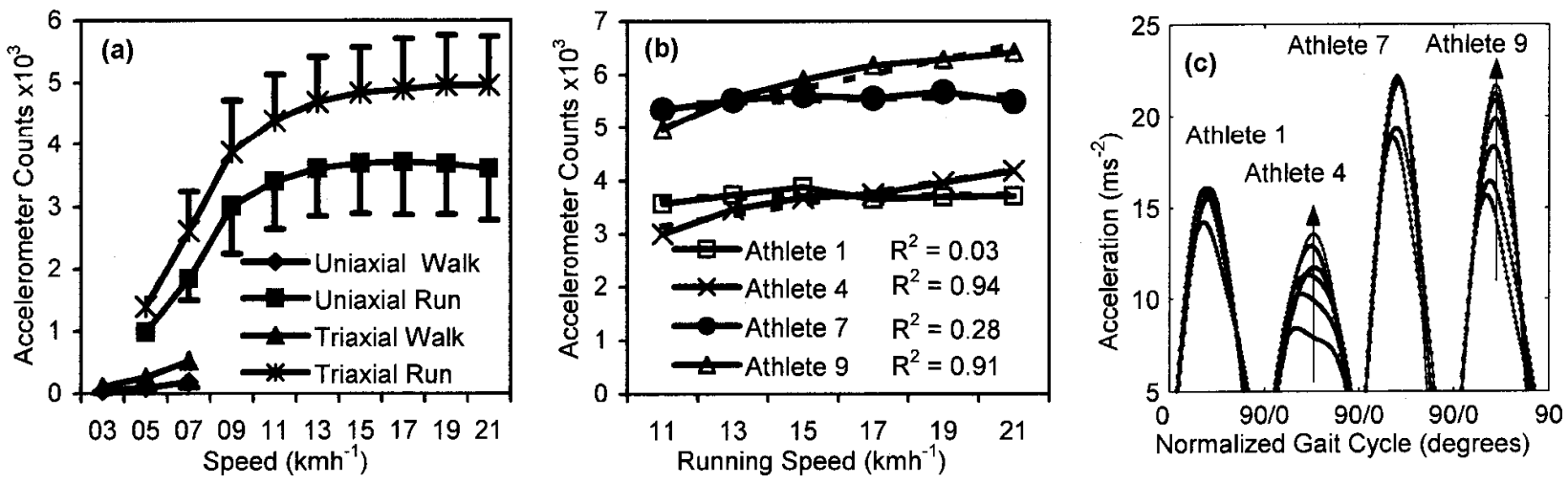

Fig. 5. (a) Vertical-uniaxial and triaxial accelerometer counts for walking and running with \pm 1SD bars. (b) Individual results for athletes with the highest and lowest triaxial accelerometer counts. Dashed trend lines are included in the figure. (c) Gait cycle normalized, multispeed, vertical acceleration signal for the four athletes in (b) (left foot only). $X$-axis degrees indicate the proportion of stride cycle (complete left/right step combination $=360$ degrees). For athletes 1 and 7 , the magnitude was consistent across all running speeds (with occasional outlying traces). For athletes 4 and 9 , the magnitude consistently increased as running speed increased. The arrows indicate the change in the acceleration as running speed increased (athletes 4 and 9 only) [17].

the rating of the accelerometer-based device used in that testing. It is possible to use an infinite impulse response (IIR) filter to remove the overload spike however this form of filtering applies different attenuation factors at different step frequencies, impacting the output of the accelerometer count function. Other researchers have attempted to overcome the effects of filtering by applying step frequency-based correction factors [16].

2) Walking and running gave distinctly different signal signatures.

3) For many athletes at very low walking speeds, the step frequency was not discernable in either the time or the frequency domains.

4) Accelerometer counts from the mediolateral channel gave strong individual correlations with speed ( $R^{2}=0.91-0.99$, average $R^{2}=0.96$, group $R^{2}=0.63$ ). This did not appear to be recorded in the literature. Although this appears useful, the horizontal plane accelerometers are highly susceptible to misalignment errors due to gravity.

\section{B. Analysis of Accelerometer Results}

From Fig. 5(b), individual variations in both slope and magnitude were observed. As the predominant source of signal power is the vertical accelerometer, the acceleration signal for this channel was investigated. An FIR-H filter was used to remove signal above twice the step frequency, multiple left/right step pairs were averaged at each speed and gait cycle normalization was used to directly compare signals from different step frequencies. The output of this processing Fig. 5(c), identified characteristics of the vertical acceleration that corresponded with the slope of the accelerometer counts of Fig. 5(b). The two athletes of Fig. 5(b), with relatively flat accelerometer counts, athletes 1 and 7, exhibited consistent vertical acceleration across all running speeds in Fig. 5(c) whereas athletes 4 and 9 , exhibited both increased vertical acceleration and increased accelerometer counts as running speed increased. The slope of the Fig. 5(b) regression lines was therefore an indicator of this characteristic. It should be noted that while the change in magnitude in vertical acceleration may account for the slope of the triaxial accelerometer counts, the biomechanics of athletes 4 and 9 is such that the contribution of the medio-lateral and anterior-posterior activity causes the triaxial counts of 4 and 9 to exceed those of athletes 1 and 7 even though the vertical acceleration does not.

In the following analysis of running data, $9 \mathrm{kmh}^{-1}$ was used as a reference point as all athletes were using a running action by this speed.

Fourier analysis of the running signal identified that for most test subjects, almost the entire signal from the vertical channel was located at the step frequency. Assuming the signal was sinusoidal, the following equations applied:

$$
\begin{aligned}
& s_{t}=A \sin (2 \pi f t) \\
& a_{t}=-A(2 \pi f)^{2} \sin (2 \pi f t)
\end{aligned}
$$

where $A$ is the maximum amplitude of the signal, $f$ is the step frequency, $s_{t}$ is the displacement as a function of time, and $a_{t}$ is the acceleration as a function of time.

Based on this assumption, acceleration and accelerometer counts were functionally related to the physical displacement of the athlete's body and the step frequency. Normalizing 9 $\mathrm{kmh}^{-1}$ accelerometer counts by the average $9 \mathrm{kmh}^{-1}$ step frequency using (3) gave an estimated relative displacement factor Fig. 6(a). Referring to this factor as displacement was a matter of convenience and no separate measure was performed to confirm this estimate. This result did indicate that the acceleration counts were proportional to a factor other than step-frequency. A comparison of the average magnitude of the 9-21 kmh ${ }^{-1}$ triaxial accelerometer counts of Fig. 5(b) to the $9 \mathrm{kmh}^{-1}$ step-frequency appears in Fig. 6(d). This indicated a strong relationship between the $9 \mathrm{kmh}^{-1}$ step frequency and the overall magnitude of the accelerometer count results

$$
\mathrm{RD}_{a}=\frac{\operatorname{counts}_{a}}{\text { mean }(\text { counts })} \times\left(\frac{\operatorname{mean}(f)}{f_{a}}\right)^{4} .
$$

All values are for the designated speed $\left(9 \mathrm{kmh}^{-1}\right) . \mathrm{RD}_{a}$ is the relative displacement of athlete " $\mathrm{a}$," $f_{a}$ is the step frequency for athlete "a." The step-frequency factor used the power of 4 as acceleration (2) used the square of the frequency and counts, 

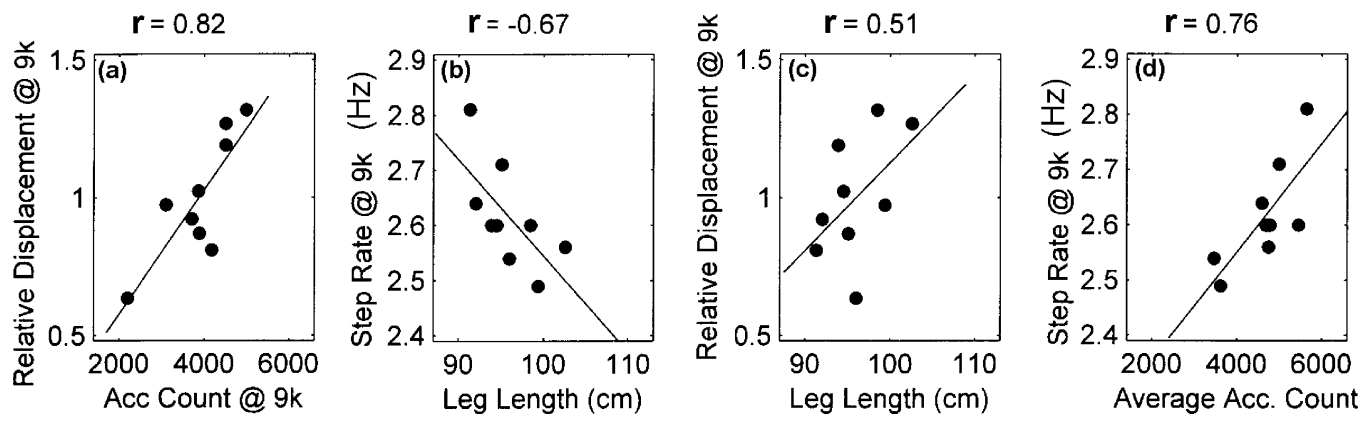

Fig. 6. Scatter plots and regression analysis for (a) relative estimated vertical displacements at $9 \mathrm{kmh}^{-1}$ (after normalizing for step frequency) versus accelerometer counts; (b) step frequency at $9 \mathrm{kmh}^{-1}$ versus leg-length; (c) relative vertical displacement at $9 \mathrm{kmh}^{-1}$ (after normalizing for step frequency) versus leg-length; and (d) step frequency at $9 \mathrm{kmh}^{-1}$ versus average running accelerometer counts [17].

as used in this analysis, used the square of acceleration over a second.

1) Summary of Accelerometer Data Analysis: Accelerometer counts for running speeds were affected by the following:

1) initial running step frequency,

2) physical displacement (assumed),

3) an individual biomechanical factor, estimated by the slope of the accelerometer counts versus speed regression line.

\section{Anthropometric and Biomechanical Analysis}

The relationship between submaximal energy expenditure, speed, and the mass of an athlete is well understood with regression analysis returning correlation coefficients similar to those observed in this study $\left(R^{2}=0.96\right)$. This phase of the investigation analyzed the effect of anthropometric factors of leg-length and mass on the step-frequency and relative displacement of the athlete.

From regression analysis, body mass did not appear to affect the initial step frequency, the $9 \mathrm{kmh}^{-1}$ relative displacement, or the slope of the accelerometer count regression line. However, mass did affect the slope of the individual's step-frequency versus speed regression lines with a correlation coefficient of $r=0.57$.

Leg length had a negative correlation to $9 \mathrm{kmh}^{-1}$ step frequency $(r=-0.67$, Fig. 6(b)) and to the slope of the accelerometer count regression line for running $(r=-0.76)$. Conversely, leg length had a positive correlation to estimated $9 \mathrm{kmh}^{-1}$ relative displacement $(r=0.51$, Fig. 6(c)). The combined effect of a negative correlation of leg length on step-frequency and a positive correlation to displacement is confounding. Presumably, longer legs tend to reduce step-frequency and at the same time increase displacement. Applying these results to Fig. 5(c), the relatively consistent amplitude of the vertical acceleration signals of athletes 1 and 7, was attributable to their longer legs. The steadily increasing acceleration of athletes 4 and 9 may be attributable to their shorter legs. The overall magnitude of the triaxial acceleration counts of Fig. 5(b) appeared to be primarily governed by initial step-frequency.

Considering the influence of leg length on various biomechanical factors with the effect of mass on step-frequency and energy, various predictor equations were tested. A simple linear predictor incorporating leg length, step-frequency, and mass resulted in a strong group correlation with the energy estimated

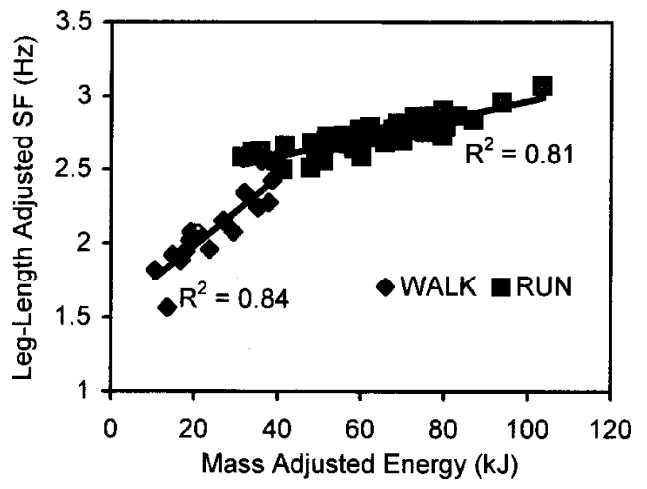

Fig. 7. Normalized leg-length adjusted step-frequency versus normalized mass adjusted energy, with trend lines and correlation coefficients [17].

from $\mathrm{VO}_{2}$. This predictor gave a group correlation coefficient for running of $R^{2}=0.81$ (Fig. 7), a large improvement from $R^{2}=0.29$. It should be noted that leaving mass out of the predictor gave a step-frequency, leg length, and $\mathrm{VO}_{2}$ energy estimate correlation of $R^{2}=0.79$.

\section{Summary of Physiological, Biomechanical, Anthropometric, and Accelerometer Signal Analysis}

For ambulatory activity, leg-length, mass, and natural stepfrequency combined to generate a good submaximal energy estimator. As two of these factors are constants, signal processing only needs to determine step-frequency. Since accelerometer counts have been shown to give a linear response at walking speeds and for low-intensity activities, a combined approach is possible. Table I indicates a software table-based approach to the processing required.

\section{Embedded System Signal Processing}

While the foregoing discussion deals specifically with extracting the energy estimator, the total system incorporates additional functionality such as contact/tackle and orientation information. For this analysis, orientation was derived using a comparison of the output of the $0.9-\mathrm{Hz}$ low-pass filter and the "steady-state" orientation. Athlete contacts appear as a band of intensity of accelerometer counts above the running band. The necessary processing is a small extension of the table driven approach and is indicated in Table I. High-intensity accelerometer 
TABLE I

ENERGY ESTIMATION AND ACTIVITY CLASSIFICATION [17]

\begin{tabular}{|l|l|l|l|l|}
\hline \multirow{2}{*}{$\begin{array}{c}\text { Triaxial } \\
\begin{array}{c}\text { Accelerometer } \\
\text { Counts }\end{array}\end{array}$} & \multicolumn{4}{|c|}{ Activity Type } \\
\cline { 2 - 5 } & Stationary & Walking & Running & Other $^{a}$ \\
\hline Below walking band & $\mathbf{X}$ & & & \begin{tabular}{l} 
Check $_{\text {angle }^{\mathrm{b}}}$ \\
\hline Walking Band
\end{tabular} \\
\hline Running Band & & $\begin{array}{l}\text { Use Acc. } \\
\text { Counts }\end{array}$ & \\
\hline Above Running Band & & & $\begin{array}{l}\text { Measure } \\
\text { Step } \\
\text { Frequency }\end{array}$ & \\
\hline
\end{tabular}

a. Other includes various football code specific activities such as tackles, marks, jumping etc.

b. Orientation combines with activity intensity to determine activity type

counts indicate activities such as jumping, high intensity in conjunction with off-normal angle indicates hard contact, near-horizontal angle and low-intensity activity indicates the player is down on the ground.

\section{A. Signal Processing Simplifications}

The largest contributors to the processing cost of this system were sample rate, the triaxial processing, and the FIR-H filter. Previously collected $150-\mathrm{Hz}$ data sets were reprocessed using sample rates of $30,25,20$, and $15 \mathrm{~Hz}$ in conjunction with different approaches to filtering. The system outputs were analyzed to ascertain if the results were consistent with the original system. Where possible, calculations were performed as integer calculations with the sample data, after offset removal, handled as 8-bit data.

Required Outputs:

- Accelerometer Counts

- Step Frequency

- Instantaneous and Steady State Orientation

In addition to the FIR-H filter, reprocessing was performed using two other systems. In the first case, the FIR-H orientation filter was replaced using a first-order lag calculation. In the second case, the FIR-H filter was replaced with a rectangular FIR (rolling average) filter (FIR-R) to extract the orientation signal. To minimize RAM memory requirements, the FIR-R filter length was of approximately 2 -s duration. This filter had a very low processing cost compared to the FIR-H filter. A long duration first-order lag was used to estimate steady-state orientation.

\section{B. Results From Simplified Processing}

In all cases, regression analysis gave a worst case $(15-\mathrm{Hz})$ correlation coefficient for triaxial accelerometer counts versus walking of $R^{2}=0.79$. Higher sampling rates improved this result. Uniaxial results gave a worst case $(15-\mathrm{Hz})$ correlation coefficient of $R^{2}=0.57$. In each reprocessing test, the $3 \mathrm{kmh}^{-1}$ uniaxial accelerometer counts were tightly clustered with the results spreading as speed increased to $7 \mathrm{kmh}^{-1}$. Clearly distinguishable thresholds between stationary, walking, running, and impacts were still evident.

Step-frequency resolution decreased significantly as sample rate decreased. This limitation could be partly resolved by averaging the frequency estimate of successive steps. The original algorithm estimated step-frequency using the integer count of samples between successive positive zero crossings. As an alternative to averaging, the zero-crossing detection algorithm was modified to provide a linear interpolation of the zero-crossing point. The separation between successive zero crossings was then reported with a fractional component. At $15-\mathrm{Hz}$ sampling, the modified algorithm gave step-frequency estimates very closely approximating the original $150-\mathrm{Hz}$ results. As sprint step frequencies are higher than those of the running speeds tested, a sample rate of $24 \mathrm{~Hz}$ was seen as a more appropriate sample rate. A beneficial effect of reduced sample rates was the ability to simplify step-detection processing.

Orientation estimations from the alternate filters degenerated with reduced maximum angle values and increased noise. This was not a serious limitation and the noise value did not approach any decision threshold. The reduced maximum values still exceeded the appropriate decision threshold. As a negative side effect of the lower quality output of the orientation filter, the accelerometer counts output increased considerably during contact and during significant changes in orientation.

Processing in both the FIR-R and first-order lag-based systems saw a reduction in the overlap between accelerometer counts and orientation angle during player contacts. This makes the differentiation between high-intensity activities such as jumping and contact less precise.

Compared to uniaxial, triaxial accelerometer counts give improved results, although this may not necessarily translate into better system results. The vertical accelerometer is the main signal source, the source of the step-frequency information and, while the athlete is upright, includes the $9.8 \mathrm{~ms}^{-2}$ signal due to gravity. Almost all the required information can be derived from the vertical accelerometer alone; however, in the absence of the FIR-H filter the additional cost of triaxial processing is not a concern.

\section{Complete Embedded Processing System}

The complete energy expenditure estimate signal processing, comprises three accelerometers sampled at $24 \mathrm{~Hz}$ with data stored in a 44 byte by three-channel circular buffer. The buffer holds approximately $1.9 \mathrm{~s}$ of data and forms the basis of an FIR-R orientation filter. The oldest value in the buffer is subtracted from a rolling average calculator prior to overwriting it with the latest sensor value. All subsequent processing occurs with an built-in 0.9-s latency using the 23rd sample back from the newest sample.

The above triaxial sample value is used to generate or detect: 1) steady-state triaxial orientation using a long duration firstorder lag, 2) current dynamic acceleration by subtracting the orientation filter value, 3) triaxial accelerometer counts, and 4) negative to positive zero crossings on the vertical axis.

Accelerometer counts are summed in 0.25-s blocks (six samples) and 1-s blocks and compared to threshold values stored in a table. Angle is monitored on the vertical channel by comparing the FIR-R output to the steady-state first-order lag output. Angles are only calculated if the accelerometer counts or the vertical orientation signal exceeds preset thresholds. Step-frequency is calculated if indicated by the triaxial accelerometer counts. 


\section{Results}

With no comparable system performing the same analysis, results have been assessed using comparisons to video data, physiological and biomechanical data from treadmill and track running, as well as comparison to manually categorized football data.

Generated step-frequencies from different activities matched those calculated manually. Discrepancies existed between the system output and the manually categorized data. This was identified and attributed to operator delay, operator error, and operator choices, for instance; short duration changes of athlete activity tended to be ignored by the operator.

This signal processing is considerably less intensive than the original system allowing the operating frequency of the microprocessor to be dropped to a few hundred kilohertz with a subsequent reduction in current draw.

Note that this system is designed for open field sports, it is not suitable for monitoring court-based sports such as basketball and tennis, where run lengths are short and there is high lateral acceleration and strenuous upper body activity. Thus, properly calibrated for individual athletes the techniques and instrumentation described provide a reliable means of determining athlete energy

\section{CONCLUSION}

The investigation and analysis of the variance of accelerometer-count based energy estimates identified mechanical, biomechanical, and anthropometrical influences. By modifying the signal processing, a combined counts and step-frequency energy estimator can be extracted which has a higher immunity to detrimental influences and which, when combined with anthropometric measures, greatly improve the energy estimates gathered for core athlete activity.

\section{REFERENCES}

[1] H. J. Montoye, H. C. G. Kemper, W. H. M. Saris, and R. A. Washburn, Measuring Physical Activity and Energy Expenditure. Champaign, IL: Human Kinetics, 1996, pp. 34-41.

[2] B. E. Ainsworth et al., "Compendium of physical activities: Classification of energy costs of human physical activities," Med. Sci. Sports Exerc., vol. 25, no. 1, pp. 71-80, 1993.

[3] J. E. Coates and F. Meade, "The energy expenditure and mechanical energy demand in walking," Ergonomics, vol. 36, pp. 97-119, 1960.

[4] J. B. Reswick, J. Perry, and D. Antonelli, "Preliminary evaluation of the vertical acceleration gait analyser (VAGA)," in Proc. 6th Annи. Symp. External Control Human Extremities, Dubrovnik, Yugoslavia, Aug./ Sep. 1978, pp. 305-314.

[5] H. J. Montoye et al., "Estimation of energy expenditure by a portable accelerometer," Med. Sci. Sports Exerc., vol. 15, pp. 403-407, 1983.

[6] G. A. King, N. Torres, C. Potter, T. J. Brooks, and K. J. Coleman, "Comparison of activity monitors to estimate energy cost of treadmill exercise," Med. Sci. Sports Exerc., vol. 36, no. 7, pp. 1244-1251, 2004.

[7] A. Yngve, A. Nilsson, M. Sjostrom, and U. Ekelund, "Effect of monitor placement and of activity setting on the MTI accelerometer output," Med. Sci. Sports Exerc., vol. 35, no. 2, pp. 320-326, 2003.

[8] S. M. Powell, D. I. Jones, and A. V. Rowlands, "Technical variability of the RT3 accelerometer," Med. Sci. Sports Exerc., vol. 35, no. 10, pp. 1773-1778, 2003.

[9] S. M. Powell and A. V. Rowlands, "Intermonitor variability of the RT3 accelerometer during typical physical activities," Med. Sci. Sports Exerc., vol. 36, no. 2, pp. 324-330, 2004.
[10] M. S. Treuth et al., "Defining accelerometer thresholds for activity intensities in adolescent girls," Med. Sci. Sports Exerc., vol. 36, no. 7, pp. 1259-1266, 2004.

[11] ADXL202 Data Sheet Analog Devices, 2006 [Online]. Available: http://www.analog.com/en/prod/0\%2C2877\%2CADXL202\%2C00. html

[12] A. Lai, D. A. James, J. P. Hayes, and E. C. Harvey, "Semi-automatic calibration technique using six inertial frames of reference," Proc. Int. Soc. Opt. Eng. (Perth, Australia), vol. SPIE 5274, pp. 531-542, Dec. 2003.

[13] A. Wixted, D. James, and D. Thiel, "Low power operating system and wireless networking for a real time sensor network," in Proc. IEEE ICITA, Bathurst, Australia, Nov. 2002, 112.

[14] D. A. James, N. Davey, and T. Rice, "An accelerometer based sensor platform for insitu elite athlete performance analysis," in Proc. IEEE Sensors Conf., Vienna, Austria, Oct. 2004, pp. 1373-1376.

[15] P. U. Saunders, R. D. Telford, D. B. Pyne, R. B. Cunningham, C. J. Gore, A. G. Hahn, and J. A. Hawley, "Improved running economy in elite runners after 20 days of simulated moderate-altitude exposure," $J$. Appl Physiol. 96, pp. 931-937, 2004.

[16] S. Brage, N. Wedderkopp, P. W. Franks, L. B. Andersen, and K Froberg, "Reexamination of validity and reliability of the CSA monitor in walking and running," Med. Sci. Sports Exerc., vol. 35, no. 8, pp. 1447-1454, 2003.

[17] A. Wixted, D. V. Thiel, A. Hahn, C. Gore, D. Pyne, and D. A. James, "Signal processing for estimating energy expenditure of elite athletes using triaxial accelerometers," in Proc. IEEE Sensors Conf., Irvine, CA, 2005, pp. 798-801.

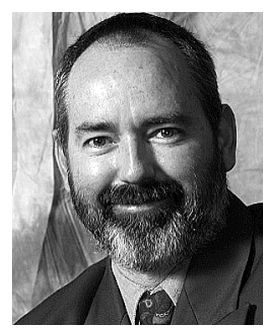

Andrew J. Wixted (S'05) received the B.Eng degree (first class honors) from the University of Southern Queensland, Toowoomba, Australia, in 1997.

$\mathrm{He}$ is currently working towards the Ph.D. degree at Griffith University, Brisbane, Australia, with his research studies focused on real-time extraction of biomechanical and physiological parameters from elite athletes engaged in training and competition.

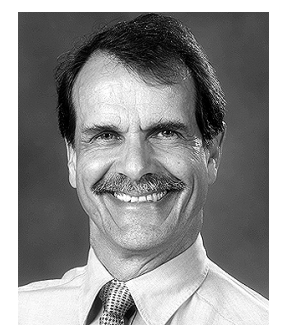

David V. Thiel (M'81-SM'88) received the B.Sc degree from the University of Adelaide, Adelaide, Australia, in 1970, and then completed the M.Sc. and Ph.D. degrees from James Cook University, Townsville, Australia, in 1974 and 1980, respectively.

He is currently Professor and Head of the Griffith School of Engineering, and Director of the Centre for Wireless Monitoring and Applications at Griffith University. He worked with the team that developed the world's first odor sensing robot. He lead the development of TSIM, a surface impedance meter used in Central Queensland coalfields for pre-mining coal seam assessment. His current research interests lie in smart antennas, numerical modeling in electromagnetics, electromagnetic geophysics, and integrated sensor and packaging technologies.

Prof. Thiel is a Fellow of the Engineers Australia. He serves as Chair of the Wave Propagation Standards Committee of the IEEE Antennas and Propagation Society and is a member of the Antennas Standards Committee.

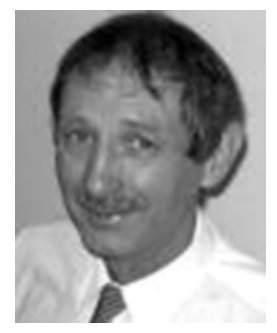

Allan G. Hahn received the Ph.D. degree from the University of Western Australia in 1986.

For the past 21 years, he has worked for the Australian Institute of Sport, including 11 years as Head of Physiology Department, prior to his current position as Head of Applied Research Centre. His achievements include: 72 publications in peer-reviewed scientific journals; 12 book chapters and technical reports; more than 30 papers in the applied sports science and coaching literature; and over $\$ 3.5$ million in research grants. 
Dr. Hahn received the National Sports Research Award for contribution to development of sport in Australia through conduct of applied research, was Appointment as Adjunct Professor in the Faculty of Science at the University of the Sunshine Coast, Queensland. He received the Australian Sports Medal in 2000 and Order of Australia Medal in 2002. He was listed by The Bulletin magazine as one of the "Smart 100" Australians, and received inaugural prize for innovation and creativity in the Sport category. He was elected Honorary Research Fellow of the Queensland Academy of Sport.

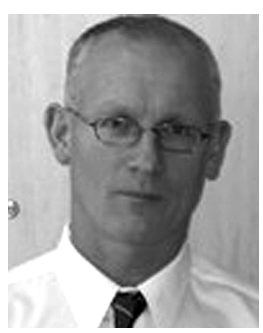

Christopher J. Gore received the Ph.D. degree from Flinders University of South Australia in 1989.

Since graduating, he has worked mostly for the Australian Institute of Sport, from 1992 until now. For the first 10 years, he established an Australia-wide, world-class quality assurance scheme for the fitness testing of Australia's elite athletes; and between 2001 and 2005, he was the manager for the Australian Institute of Sport's collaboration with micro-technology organization. For the last year he has been Head of the Department of Physiology. He is internationally recognized as an expert in altitude training and as a scientist he has more than 90 publications in peer-reviewed journals and over 100 conference presentations.

Dr. Gore was awarded a Sport Australia Medal for his contribution to the team that developed a blood-based test for EPO that was implemented at the Sydney Olympic Games in 2000. In 2006, he received one of the 40 inaugural Distinguished Alumni awards from Flinders University (those 40 were selected from approximately 50000 graduates). Most recently, he was also awarded a Professorial fellowship at Flinders University.
David B. Pyne received the M.A.ppSc. degree from the University of Canberra, Canberra, Australia, in 1989, and the Ph.D. degree from the Australian National University, Canberra, in 1995.

$\mathrm{He}$ is a sports scientist in the Department of Physiology at the Australian Institute of Sport (AIS). His work in the areas of the applied physiology of swimming, exercise, and the immune system, and fitness and conditioning for team sports has been recognized internationally. He has published extensively in both the scientific peer-reviewed literature and lay articles for the broader sporting community: 96 peer-reviewed full papers, 72 peer-reviewed abstracts, and 126 lay or coaching publications. He holds honarary appointments as an Adjunct Associate Professor, Medical School, at the Australian National University, and as a Professional Associate in the Division of Science, Health and Design at the University of Canberra. He is the Foundation Editor of the International Journal of Sports Physiology and Performance.

Dr. Pyne received the Australian Sports Medal in 2000 and an Australia Day Achievement Medallian in 2003. He is a member of Sports Medicine Australia, and a Fellow of the American College of Sports Medicine.

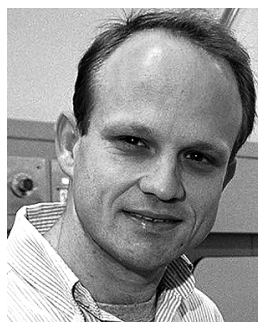

Daniel A. James (M'93-SM'06) received the Ph.D. degree from Griffith University, Brisbane, Australia, in 1998 after receiving the B.Sc. and M.Phil. degrees there.

He is currently a Senior Research Fellow with the Centre for Wireless Monitoring and Applications at Griffith University. His principle areas of research intrest are in applying sensors to sporting and biomedical applications. He has around 50 publications in this area including several international patents. 